\title{
Invasive nontyphoidal Salmonella infection in a patient with Roux-en-Y gastric bypass and gastrostomy tube: a case report
}

\author{
Luxi Chen ${ }^{1}$, Temitayo Olojo ${ }^{2}$ and Bader Aldeen Alhafez ${ }^{2^{*}}$ (i)
}

\begin{abstract}
Background: Infections with nontyphoidal Salmonella most commonly cause a self-limited gastroenteritis in humans and are the leading source of foodborne illness. In the USA, the incidence of culture-confirmed invasive Salmonella is extremely rare. Here, we present a unique case of enteric nontyphoidal Salmonella enteritidis infection that progressed to an invasive bacteremia in a patient with Roux-en-Y gastric bypass and gastrostomy feeding tube.

Case presentation: A 58-year-old Caucasian woman with a past medical history of Roux-en-Y gastric bypass with revision and recent gastrostomy feeding tube insertion was admitted with fever and diarrhea. During her inpatient stay, two sets of blood cultures grew Salmonella enteritidis and she was promptly treated with intravenous antibiotics. Her hospital course was also complicated by acute kidney injury. The patient recovered after treatment with a thirdgeneration cephalosporin and supportive treatment.

Conclusion: We present an unusual case of nontyphoidal Salmonella enteritidis bacteremia complicated by acute kidney injury in a middle-aged woman with a previous history of Roux-en-Y gastric bypass and gastrostomy feeding tube insertion. Further investigation is needed to identify whether gastric surgeries or procedures are independent risk factors for invasive nontyphoidal salmonellosis.
\end{abstract}

Keywords: Nontyphoidal Salmonella, Bacteremia, Bariatric surgery

\section{Introduction}

Salmonellae are a genus of motile, facultative intracellular Gram-negative bacilli that belong to the Enterobacteriaceae family [1]. As one of the most common causes of foodborne illness, Salmonella infection is a major global public health concern. Salmonella is transmitted to humans via the fecal-oral route, often through contaminated foods of animal origin such as poultry, beef, fish, milk, and eggs. Salmonella is divided into two subspecies, nontyphoidal and typhoidal, that are genetically similar

\footnotetext{
*Correspondence: bdalhafez@gmail.com

${ }^{2}$ Department of Internal Medicine, Division of Hospital Medicine, The

Ohio State University Wexner Medical Center, M112 Starling Loving Hall, 320 West 10th Avenue, Columbus, OH 43210, USA

Full list of author information is available at the end of the article
}

but cause distinct disease states and immune responses in humans. Nontyphoidal serovars typically cause a selflimiting gastrointestinal (GI) disease, while typhoidal serovars cause enteric or typhoid fever, an invasive, systemic, and life-threatening disease that results in more than 200,000 deaths annually worldwide [2].

Nontyphoidal Salmonella are a leading cause of bacterial diarrhea. Although the majority of nontyphoidal Salmonella infections result in a self-limiting, mild-tomoderate gastroenteritis with low fatality, approximately $1-4 \%$ of enteric infections lead to bacteremia [3]. The greatest burden of nontyphoidal Salmonella bacteremia is present in Sub-Saharan Africa, accounting for $79 \%$ of the total number of cases globally [4]. Causes of serious GI infections may include overuse of antimicrobials, radiation, and immunocompromised states such as original author(s) and the source, provide a link to the Creative Commons licence, and indicate if changes were made. The images or other third party material in this article are included in the article's Creative Commons licence, unless indicated otherwise in a credit line to the material. If material is not included in the article's Creative Commons licence and your intended use is not permitted by statutory regulation or exceeds the permitted use, you will need to obtain permission directly from the copyright holder. To view a copy of this licence, visit http://creativecommons.org/licenses/by/4.0/. The Creative Commons Public Domain Dedication waiver (http://creativeco mmons.org/publicdomain/zero/1.0/) applies to the data made available in this article, unless otherwise stated in a credit line to the data. 
cancer and obesity [5-7]. A high incidence of invasive disease occurs in children younger than 5 years of age, the elderly, and immunocompromised individuals, particularly those with human immunodeficiency virus (HIV) infection. In the USA, the mean population incidence of culture-confirmed invasive Salmonella is rare, roughly 0.9 cases per 100,000 people annually, mostly occurring in infants [8]. Despite its rarity, invasive Salmonella bacteremia typically requires hospitalization as it can be severe and life-threatening [9].

Here, we present an unusual case of nontyphoidal Salmonella infection that progressed from enteric to invasive bacteremia in an adult patient with a previous history of Roux-en-Y gastric bypass. Our patient lacked the classic predisposing risk factors for this invasive infection, namely extremes of age, chronic liver disease, and immunosuppressing conditions [10]. We discuss less common risk factors that could alter the GI tract's microenvironment, such as gastric surgeries and chronic proton pump inhibitor (PPI) use, as they could contribute to the progression from enteric to systemic Salmonella bacteremia. Therefore, these risk factors should be considered in the evaluation of the appropriate patient population.

\section{Case presentation}

A 58-year-old Caucasian female presented to her local emergency department with fever and diarrhea. The patient developed nonbloody, large-volume diarrhea after eating take-out 11 days ago, with four to five bowel movements per day. During this time, she also experienced fevers, chills, nausea, vomiting, general malaise, and weakness. In addition, she observed leaking and drainage around her G-tube site with associated erythema and intense abdominal pain. The patient denied sick contacts or recent travel.

Her past medical history includes obesity, orthostatic hypotension, hyperlipidemia, chronic kidney disease, nephrolithiasis, gastrojejunal ulcer, Kawasaki's disease, subdural hemorrhage, epilepsy, chronic pain syndrome, and anxiety. The patient's past surgical history includes burr-hole craniotomy, lithotripsy, cholecystectomy, Roux-en-Y gastric bypass complicated by chronic refractory marginal ulceration and small bowel obstruction requiring two resections, ventral hernia repair, and gastrostomy tube insertion.

The patient was pregnant six times and had six miscarriages (G6P0060). She lives with her husband and adopted son with good social support. She is currently unemployed. The patient denies ever smoking and does not drink alcohol. Familial history consists of diabetes, heart disease, and nephrolithiasis in the patient's mother. Her father had glaucoma, nephrolithiasis, and prostate cancer.
Medications before admission included pantoprazole $40 \mathrm{mg}$ twice a day, sucralfate $1 \mathrm{~g}$ twice a day, ondansetron $4 \mathrm{mg}$ twice a day as needed for nausea, midodrine $10 \mathrm{mg}$ three times a day, fludrocortisone $0.1 \mathrm{mg}$ twice a day, atorvastatin $40 \mathrm{mg}$ daily, levetiracetam 1500 twice a day, methadone $10 \mathrm{mg}$ every 8 hours, and lorazepam $0.5 \mathrm{mg}$ daily as needed for anxiety. She was also on daily vitamin A, D3, and B-complex supplements and a monthly cyanocobalamin $1000 \mu \mathrm{g}$ intramuscular injection, in addition to a nutritional supplement Isosource $1.5 \mathrm{Cal}$ that is administered through the G-tube once every night.

Due to her complex past surgical history, the patient was sent from her local emergency department to our hospital. At presentation, the patient was febrile to $101^{\circ} \mathrm{F}$. Blood pressure was $110 / 72 \mathrm{mmHg}$, and heart rate was 84 beats per minute. Further examination revealed diffuse abdominal tenderness with erythema and purulent drainage around the G-tube site on the left upper quadrant. The general examination showed a thin patient who is alert and oriented. Mucous membranes were moist. Lungs were clear to auscultation as she was breathing comfortably on room air. Heart sounds were regular without murmurs. Extremities were warm with intact pulses bilaterally and no edema. However, she had moderate muscle loss for her age. Skin was intact except for the previously mentioned erythema around the G-tube. Neurological examination revealed normal speech with comprehension, intact cranial nerves II-VII, XI, and XII, and 5/5 motor strength in all four extremities. Sensation was intact to light touch and pinprick throughout. Romberg test was negative. Gait was slowed owing to weakness but showed normal balance.

Serum chemistry showed sodium of $136 \mathrm{mmol} / \mathrm{L}$, potassium of $2.9 \mathrm{mmol} / \mathrm{L}$, chloride of $111 \mathrm{mmol} / \mathrm{L}$, bicarbonate of $20 \mathrm{mmol} / \mathrm{L}$, magnesium $1.2 \mathrm{mg} / \mathrm{dL}$, calcium $6.1 \mathrm{mg} / \mathrm{dL}$, and serum creatinine $1.0 \mathrm{mg} / \mathrm{dL}$. Liver function tests showed low albumin of $2.5 \mathrm{~g} / \mathrm{dL}$, total bilirubin of $0.3 \mathrm{mg} / \mathrm{dL}$, alkaline phosphatase of $86 \mathrm{U} / \mathrm{L}$, alanine aminotransferase (ALT) of $13 \mathrm{U} / \mathrm{L}$ and aspartate aminotransferase (AST) of $21 \mathrm{U} / \mathrm{L}$. Complete blood count showed a white blood cell count of $3.97 \times 10^{9} / \mathrm{L}$, hemoglobin of $12.6 \mathrm{~g} / \mathrm{dL}$, hematocrit of $39.6 \%$, and platelet count of $239 \times 10^{9} / \mathrm{L}$. Urinalysis showed normal appearance and color, specific gravity of 1.020, without detecting red blood cells (RBC), white blood cells (WBC), ketones, glucose, leukocytes esterase, or nitrites. A stool sample was sent for Clostridium difficile and a molecular enteric panel but was pending at that time. Two out of two blood cultures that were collected at her local emergency department were positive for Gram-negative rods. An abdominal radiograph confirmed G-tube location overlying the stomach and a computed tomography (CT) abdomen/pelvis with contrast showed nonspecific 
skin thickening and subcutaneous fat stranding along the G-tube insertion site (Fig. 1). Due to a prior history of penicillin and sulfa anaphylactic reactions, the patient was empirically treated with intravenous ciprofloxacin $400 \mathrm{mg}$ every 8 hours for bacteremia, and oral clindamycin $450 \mathrm{mg}$ every 8 hours for the cellulitis surrounding her G-tube site, in addition to intravenous fluid resuscitation with normal saline at $75 \mathrm{~mL} /$ hour and electrolyte repletion with oral potassium chloride $20 \mathrm{mEq}$ for four total doses, and intravenous magnesium sulfate $4 \mathrm{~g}$ once.

A fluoroscopy G-tube study revealed no contrast leak or evidence of obstruction. On day 1 of hospitalization, the molecular enteric stool panel was positive for the Salmonella species. On day 4, two out of two blood cultures grew Salmonella enteritidis with susceptibilities to ampicillin, ceftriaxone, and trimethoprim/sulfamethoxazole. The patient was then transitioned from intravenous ciprofloxacin to a 14-day course of intravenous ceftriaxone $2 \mathrm{~g}$ every 24 hours. We resumed tube feeds through the G-tube on day 5 after a successful clamping trial. On day 6 , the patient was somnolent and difficult to arouse, with poor skin turgor and dry mucous membranes. Vital signs remained within normal limits; $\mathrm{T}$ max of $98.6{ }^{\circ} \mathrm{F}$, blood pressure of $98 / 65 \mathrm{mmHg}$, and heart rate of 95 beats per minute. Laboratory results were significant for a serum creatinine level of $5.25 \mathrm{mg} / \mathrm{dL}$ and non-anion gap metabolic acidosis with sodium of $131 \mathrm{mmol} / \mathrm{L}$, chloride of $105 \mathrm{mmol} / \mathrm{L}$, and serum bicarbonate of $18 \mathrm{mmol} / \mathrm{L}$. These clinical and laboratory findings of dehydration suggested the development of prerenal acute kidney injury (AKI) in the setting of prolonged GI volume loss. The patient was resuscitated with half normal saline containing $75 \mathrm{mEq}$ sodium bicarbonate additive at $125 \mathrm{~mL} /$ hour. On day 8 , the patient's mental status improved but her

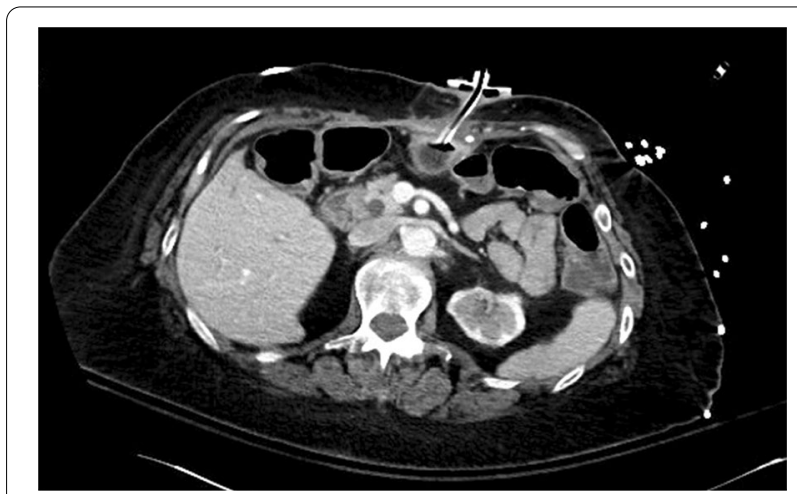

Fig. 1 Computed tomography scan of the abdomen showing Roux-en-Y gastric bypass anatomy with patent gastrojejunostomy. A percutaneous gastrostomy tube is present in the excluded portion of the stomach in an appropriate position. Some skin thickening and subcutaneous fat stranding are seen along the insertion site
G-tube began to leak, prompting clamping of the G-tube. The patient was encouraged to increase oral intake. Intravenous fluids were discontinued on day 9 , as she was able to tolerate oral foods and liquids well. No further leakage was seen around her G-tube site. On day 13, the patient was discharged home with a peripherally inserted central line to finish a 14-day course of intravenous ceftriaxone (2 g every 24 hours).

Following completion of a 14-day course of intravenous ceftriaxone, the patient made a full recovery with resolution of her diarrhea and abdominal pain. Tube feeds were resumed without further issues. At a 4-month follow-up visit with her bariatric surgeon, the patient reports that she is doing well and happy with her progress. Electrolytes and serum creatinine were all within normal limits. The patient was readmitted 6 months following her initial hospitalization for abdominal pain and hematemesis due to a large marginal ulcer. She improved with conservative measures including bowel rest (nothing per os) and maintenance intravenous fluids, and was discharged 2 days later. At the time of discharge, her hemoglobin remained stable at $11.2 \mathrm{~g} / \mathrm{dL}$, and she was able to tolerate an oral diet. She continues to follow up with her bariatric surgeon in clinic with no recurrence of fever or diarrhea.

\section{Discussion}

In summary, we present a unique case of nontyphoidal Salmonella enteritidis bacteremia complicated by acute kidney injury in a middle-aged woman with a previous history of Roux-en-Y gastric bypass and G-tube insertion. The patient initially developed Salmonella gastroenteritis through a fecal-oral route, which progressed to invasive bacteremia likely secondary to alterations of the GI tract and malnutrition. She was successfully treated with a 14-day course of intravenous ceftriaxone and fluid resuscitation.

Although the patient lacked the classic predisposing risk factors for invasive nontyphoidal Salmonella infection, namely extremes of age, chronic liver disease, malaria, and immunosuppressing conditions [10], her past medical history of Roux-en-Y gastric bypass with chronic PPI use likely altered the microenvironment of her GI tract and contributed to the progression from enteric to systemic Salmonella bacteremia. Patients who undergo gastric bypass surgery are often prescribed prophylactic proton pump inhibitors (PPIs) post-surgery to reduce marginal ulceration [11]. Chronic PPI use decreases gastric acid secretions and increases the risk of infections that are transmitted via the fecal-oral route [12]. When long-term PPI administration suppresses gastric acid secretion in the GI tract, there is a higher susceptibility to infection and proliferation of acid-labile bacteria such as Salmonella. Furthermore, recent studies 
have revealed that, within a month of PPI initiation, there is a significant decrease in the diversity of normal gut flora [13]. These alterations in the intestinal microbiome can result in the proliferation of pathogenic bacteria, leading to activation of immune cells and cytokine mediators that trigger intestinal inflammation. In the case of our patient, her chronic PPI use since undergoing bariatric surgery in 2004 likely predisposed her to GI tract infections and led to the progression of an enteric Salmonella infection to invasive bacteremia.

Interestingly, Siddiqui et al. recently reported a case in which a patient developed chronic Salmonella infection status post Roux-en-Y gastric bypass surgery that was subsequently treated successfully with laparoscopic cholecystectomy [14]. Of note, the patient in this case report had positive stool and urine cultures for Salmonella but negative blood cultures. Similarly, Shogbesan et al. described a case of Shigella sonnei bacteremia in an otherwise healthy patient with a prior history of Rouxen-Y gastric bypass [15]. The gut itself has been shown to serve as a clinically significant portal of entry for bacterial infection and bacteremia, particularly in the setting of nutritional depletion combined with exposure to endotoxins [16]. Future investigations are warranted to establish whether gastric surgeries and/or procedures independently predispose to the development of Salmonella bacteremia in the absence of other risk factors.

Several studies have shown that severe, chronic malnutrition plays an important role in the development of invasive Salmonella disease. In Sub-Saharan Africa, a distinct genotype of nontyphoidal Salmonella enterica, ST313, has adapted in malnourished patient populations to cause invasive disease [17]. In Kenya, roughly onethird of all pediatric cases of nontyphoidal Salmonella bacteremia occur in children with severe malnutrition [18]. Malnutrition has been shown to cause immunodeficiency and altered GI tract structure and function, reducing the ability of the immune system to protect against infections [19]. Indeed, by the time our patient presented to the hospital, she already experienced 11 days of severe diarrhea and poor oral intake due to nausea and vomiting. In addition, she was unable to supplement her diet through her G-tube owing to infection. These factors, along with laboratory values showing hypocalcemia, hypokalemia, and hypomagnesemia, suggest that the patient was suffering from malnutrition that possibly triggered the development of Salmonella bacteremia.

During the patient's hospital stay, she also experienced acute kidney injury with rising serum creatinine levels. Acute renal dysfunction has been described in previous studies in the setting of severe Salmonella infection and is commonly attributed to dehydration due to GI losses, shock, rhabdomyolysis, or intrinsic renal disease such as acute tubular necrosis [20]. In fact, the incidence of renal impairment accompanying Salmonella gastroenteritis is fivefold higher compared with that caused by other gastrointestinal pathogens. Overall, the prognosis of acute kidney injury as a result of Salmonella infection appears to have a benign course. A majority of currently reported cases resolved successfully with aggressive fluid replacement and appropriate antibiotic treatment without the need for hemodialysis, as with our patient [20-22]. Thus, early recognition and prompt treatment of acute kidney injury are especially important in the context of Salmonella gastroenteritis and/or bacteremia.

Salmonella enteritidis is the third most common serotype to cause culture-confirmed invasive Salmonella infections in the USA [8]. Nontyphoidal Salmonella bacteremia is treated empirically with either intravenous fluoroquinolones or third-generation cephalosporins and tailored once susceptibility data become available. The duration of antimicrobial therapy varies, with longer courses warranted for patients with significant immunosuppression [23]. While our patient was successfully treated with a 2-week course of intravenous ceftriaxone, reduced susceptibility and multidrug resistance to fluoroquinolones and third-generation cephalosporins remain a major public health issue worldwide. In Sub-Saharan Africa, multidrug resistance was observed in $75 \%$ of nontyphoidal Salmonella isolates [24]. In the USA, the number of ceftriaxone-resistant bloodstream isolates doubled from 2.5\% between 1996 and 2007 to 5\% between 2003 and 2013 [25]. Thus, sound judgment in antimicrobial use in both humans and food-producing animals is paramount to limit the spread of antibiotic resistance and emergence of more virulent strains of Salmonella.

\section{Conclusion}

In conclusion, we present an unusual case of nontyphoidal Salmonella enteritidis bacteremia complicated by acute kidney injury in an adult patient with a prior history of Roux-en-Y gastric bypass and G-tube insertion that was successfully treated with a 2-week course of intravenous antibiotics and extensive fluid resuscitation. The cause of the patient's Salmonella bacteremia was most likely multifactorial, due to an altered microbiome status post bariatric surgery, chronic PPI use, and malnutrition secondary to large volume diarrhea and poor oral intake. In patients with a Roux-en-Y gastric bypass who develop Salmonella gastroenteritis, there should be a high index of suspicion for possible systemic spread as early recognition is important to initiate appropriate management and prevent complications. Further investigation is necessary to identify whether gastric surgeries or procedures are independent risk factors for invasive nontyphoidal salmonellosis. 


\section{Acknowledgements}

The authors would like to thank the patient for her kindness and permission to report her case.

\section{Authors' contributions}

LC performed the patient's chart review, analyzed laboratory and imaging findings, did background research on the subject matter, wrote the case report, and discussed it with the corresponding author and coauthor. TO obtained written consent from the patient, analyzed data, and provided guidance on the paper. BAA analyzed data, provided expertise and guidance on the subject, and revised the paper. All authors read and approved the final manuscript.

\section{Funding}

The authors received no financial support for the study and collection, data interpretation, analysis, and writing conducted for this manuscript.

\section{Availability of data and materials}

Not applicable.

\section{Declarations}

Ethics approval and consent to participate

Written informed consent was obtained from the patient.

\section{Consent for publication}

Written informed consent was obtained from the patient for publication of this case report and accompanying images. A copy of the written consent is available for review by the Editor-in-Chief of this journal.

\section{Competing interests}

The authors report no conflicts of interest.

\section{Author details}

${ }^{1}$ Medical Scientist Training Program, The Ohio State University College of Med icine, Columbus, USA. ${ }^{2}$ Department of Internal Medicine, Division of Hospital Medicine, The Ohio State University Wexner Medical Center, M112 Starling Loving Hall, 320 West 10th Avenue, Columbus, OH 43210, USA

Received: 18 March 2021 Accepted: 6 July 2021

Published online: 05 August 2021

\section{References}

1. Jajere SM. A review of Salmonella enterica with particular focus on the pathogenicity and virulence factors, host specificity and antimicrobial resistance including multidrug resistance. Vet World. 2019;12(4):504-21.

2. Buckle GC. Typhoid fever and paratyphoid fever: systematic review to estimate global morbidity and mortality for 2010. J Global Health. 2012 https://doi.org/10.7189/jogh.02.010401.

3. Buchwald DS. A review of human salmonellosis: II. Duration of excretion following infection with nontyphi Salmonella. Rev Infect Dis. 1984;6(3):345-56

4. Stanaway JD. The global burden of nontyphoidal Salmonella invasive disease: a systematic analysis for the Global Burden of Disease Study. Lancet Infect Dis. 2017:19(12):1312-24.

5. Devaraj NK. Antibiotic resistance: a real menace. Oman Med J. 2017:32(6):531.
6. Devaraj NK, Suppiah S, Veettil SK, Ching SM, Lee KW, Menon RK, et al. The effects of probiotic supplementation on the incidence of diarrhea in cancer patients receiving radiation therapy: a systematic review with meta-analysis and trial sequential analysis of randomized controlled trials. Nutrients. 2019:11(12):2886.

7. Sook L, Sablihan N, Ismail S, Devaraj N, Mooi C. Factors associated with the level of physical activities among non-academic staffs in the Faculty of Medicine and Health Sciences of a public university in Selangor. Malaysia. 2019:15:47-55.

8. Vugia DJ. Invasive Salmonella infections in the United States, FoodNet, 1996-1999: incidence, serotype distribution, and outcome. Clin Infect Dis. 2004;38(s3):S149-56

9. Barton Behravesh C, Jones TF, Vugia DJ, Long C, Marcus R, Smith K, et al. Deaths associated with bacterial pathogens transmitted commonly through food: foodborne diseases active surveillance network (FoodNet), 1996-2005. J Infect Dis. 2011;204(2):263-7.

10. Gordon MA. Salmonella infections in immunocompromised adults. J Infect. 2008:56(6):413-22

11. Ying VWC. Prophylactic PPI help reduce marginal ulcers after gastric bypass surgery: a systematic review and meta-analysis of cohort studies. Surg Endosc. 2015;29(5):1018-23.

12. Dial MS. Proton pump inhibitor use and enteric infections. Am J Gastroenterol. 2009;104(Suppl):2.

13. Jaynes $M$, Kumar $A B$. The risks of long-term use of proton pump inhibitors: a critical review. Ther Adv Drug Saf. 2019;10:2042098618809927.

14. Siddiqui Z. A case of a chronic Salmonella infection following Roux-en-Y gastric bypass surgery, treated successfully by a laparoscopic cholecystectomy. BMJ Case Rep. 2017. https://doi.org/10.1136/bcr-2017-219395.

15. Shogbesan O, Rettew A, Shaikh B, Abdulkareem A, Donato A. Bacteremia presenting with profound hepatic dysfunction. Case Rep Gastrointest Med. 2017:2017:7293281.

16. Deitch EA, Winterton J, Li M, Berg R. The gut as a portal of entry for bacteremia. Role of protein malnutrition. Ann Surg. 1987;205(6):681-92.

17. Feasey NA. Invasive non-typhoidal Salmonella disease: an emerging and neglected tropical disease in Africa. Lancet (British edition). 2012:379(9835):2489-99.

18. Brent AJ. Salmonella bacteremia in Kenyan children. Pediatric Infect Dis J. 2006;25(3):230-6

19. Bourke CD. Immune dysfunction as a cause and consequence of malnutrition. Trends Immunol. 2016;37(6):386-98.

20. van Doorn JK. Acute renal dysfunction in Salmonella gastroenteritis. J Clin Gastroenterol. 2006:40(10):910-2.

21. Campistol JM. Rhabdomyolysis and acute renal failure associated with Salmonella enteritidis infection. J Hosp Infect. 1989;14(3):267-8.

22. Neau D. Rhabdomyolysis and Salmonella enteritidis infection. Eur J Clin Microbiol. 2000;19(12):973-5.

23. Kariuki S, Gordon MA, Feasey N, Parry CM. Antimicrobial resistance and management of invasive Salmonella disease. Vaccine. 2015;33(Suppl 3):C21-9.

24. Tack B, Vanaenrode J, Verbakel JY, Toelen J, Jacobs J. Invasive non-typhoidal Salmonella infections in sub-Saharan Africa: a systematic review on antimicrobial resistance and treatment. BMC Med. 2020;18(1):212.

25. Angelo KM. Antimicrobial resistance among nontyphoidal Salmonella isolated from blood in the United States, 2003-2013. J Infect Dis. 2016:214(10):1565-70.

\section{Publisher's Note}

Springer Nature remains neutral with regard to jurisdictional claims in published maps and institutional affiliations. 